\title{
Anatomia da escápula aplicada à abordagem cirúrgica posterior: Parâmetros de segurança durante acesso ao ângulo lateral*
}

\section{Anatomy of the Scapula Applied to the Posterior Surgical Approach: Safety Parameters during Access to the Lateral Angle}

\author{
Miguel Pereira da Costa ${ }^{1}$ André Canal Braga ${ }^{1}$ Rogério Augusto Geremias ${ }^{1}$ \\ Antonio Carlos Tenor Junior ${ }^{1}$ Fabiano Rebouças Ribeiro ${ }^{10}$ Rômulo Brasil Filho ${ }^{1}$ \\ ${ }^{1}$ Grupo de Ombro e Cotovelo, Hospital do Servidor Público Estadual
de São Paulo, São Paulo, SP, Brasil \\ Endereço para Correspondência Rogério Augusto Geremias, Grupo \\ de Ombro e Cotovelo, Hospital do Servidor Público Estadual de \\ São Paulo, São Paulo, SP, Brasil (e-mail: rogerioageremias@me.com).
}

Rev Bras Ortop 2019;54:587-590.

\section{Resumo \\ Palavras-chave \\ - escápula/anatomia \& histologia \\ - escápula/cirurgia \\ - ombro}

Objetivo O presente trabalho teve como objetivo identificar parâmetros de segurança para a realização da via de acesso cirúrgico posterior da escápula por meio de um estudo transversal em cadáveres.

Métodos Foram dissecados 13 ombros de cadáveres sem história de cirurgia ou disfunção musculoesquelética prévia e em bom estado de conservação, com médias de idade, peso e altura de 70,1 anos, $61,5 \mathrm{~kg}, 1,64 \mathrm{~m}$, respectivamente. Identificou-se marco anatômico da via estudada (tubérculo infraglenoidal) e sua distância para os nervos axilar e supraescapular foi medida.

Resultados A distância média encontrada entre o tubérculo infraglenoidal ( $\mathrm{TI}$ ) e o nervo axilar (NA) foi de $23,8 \mathrm{~mm}$ e distância média do TI ao nervo supraescapular (NSE) foi de $33,2 \mathrm{~mm}$.

Conclusão A via posterior pelo intervalo entre os músculos infraespinal e redondo menor é considerada segura; porém, é preciso atenção e cautela durante o afastamento muscular, devido à curta distância média entre o sítio de fratura e a localização do NSE e do NA. Tais precauções podem evitar maiores complicações pós-operatórias.

\begin{abstract}
Objective The objective of this paper was to identify safety parameters in the posterior surgical approach of the scapula through a cross-sectional cadaver study. Methods Thirteen cadaver shoulders with no history of surgery or prior musculoskeletal dysfunction with mean age, weight, and height of 70.1 years, $61.5 \mathrm{~kg}$, and $1.64 \mathrm{~m}$, respectively, were dissected. The anatomic landmark of the studied pathway (infraglenoid tubercle) and its distance to the axillary and suprascapular nerves were measured.
\end{abstract}

Trabalho desenvolvido no Grupo de Ombro e Cotovelo, Hospital do Servidor Público Estadual de São Paulo, São Paulo, SP, Brasil. Publicado Originalmente por Elsevier Editora Ltda. recebido

08 de agosto de, 2017 aceito

21 de Dezembro de, 2017
DOI https://doi.org/

10.1016/j.rbo.2017.12.014. ISSN 0102-3616.
Copyright $\odot 2019$ by Sociedade Brasileira License terms de Ortopedia e Traumatologia. Published by Thieme Revnter Publicações Ltda, Rio de Janeiro, Brazil 


\section{Keywords}

- scapula/anatomy \& histology

- scapula/surgery

- shoulder
Results The mean distance between the infraglenoid tubercle (IT) and the axillary nerve (AN) was $23.8 \mathrm{~mm}$ and the mean distance from the IT to the suprascapular nerve (SN) was $33.2 \mathrm{~mm}$.

Conclusion The posterior approach may be considered safe through the interval between the infraspinatus and teres minor. However, caution should be taken during muscle spacing because of the short distance between the fracture site and the location of the SN and AN. These precautions help to avoid major postoperative complications.

\section{Introdução}

A incidência de fraturas da escápula tem aumentado na prática ortopédica, são geralmente associadas a traumas de grande energia, acometem principalmente adultos jovens (35-45 anos) vítimas de acidentes automobilísticos ou quedas de grandes alturas. ${ }^{1-3}$ As indicações para o tratamento cirúrgico nas fraturas do colo da glenoide são quando se apresentam desviadas com de translação maior de um centímetro, ou com o ângulo glenopolar menor do que 20 o (normal 30。 a 45०). ${ }^{4} \mathrm{O}$ objetivo da cirurgia é restaurar o comprimento, o alinhamento, e a rotação da escápula e levar à melhoria dos resultados funcionais. $^{5}$

Abordagens estendidas à escápula oferecem excelente visualização posterior, úteis para o acesso ao corpo, à espinha e ao colo da glenoide. ${ }^{6} \mathrm{~A}$ abordagem posterior descrita por Judet implica a ampla dissecção do músculo infraespinal, foi muito usada no tratamento de fraturas escapulares, mas tem sido substituída por variações menos invasivas, devido à alta morbidade e ao risco de lesões neurovasculares. ${ }^{5}$

Na abordagem posterior, é importante identificar e proteger o nervo supraescapular (NSE), que emana da incisura espinoglenoidal para inervar o músculo infraespinal na sua fossa (tração nesse nervo pode causar fraqueza do manguito rotador e deve ser evitada). ${ }^{6}$

Apesar das variações, normalmente as abordagens menos invasivas usam o intervalo entre o músculo infraespinal (NSE) e o músculo redondo menor (nervo axilar - NA), para acessar o ângulo lateral da escápula. Com a abdução do ombro em 60 o a 90。, o acesso é facilitado devido ao afastamento superior das fibras do deltoide posterior; porém, uma abdução excessiva pode tensionar o NA e colocá-lo em risco durante o acesso e trazê-lo mais próximo do campo cirúrgico. ${ }^{7-10}$

O objetivo deste trabalho é avaliar a distância média e proximidade do NA e do NSE ao tubérculo infraglenoidal (TI) para quantificar uma zona de segurança e assim auxiliar o cirurgião durante o acesso cirúrgico ao colo e ao corpo da escápula.

\section{Métodos}

O estudo em cadáveres foi elaborado no Serviço de Verificação de Óbitos, a partir da dissecção de uma amostra total de 13 ombros de 13 cadáveres, lateralidade aleatória, no intuito de identificar as estruturas neurovasculares periescapulares
(NA e NSE) e obter as medições de suas distâncias até oTI. Foi autorizada apenas uma dissecção por cadáver.

A definição do TI como marco ósseo foi determinada devido a sua posição fixa em relação às estruturas ósseas e ser a topografia em que o NSEse torna visível diretamente na via cirúrgica em questão.

A abordagem desenvolvida foi a de Judet modificada, que se mostrou uma opção excelente para a fixação de fraturas da escápula, uma vez que proporciona ótima exposição sem agredir tanto a musculatura escapular por não envolver desinserção de suas fibras. ${ }^{5}$ Cada cadáver foi posicionado em decúbito lateral de acordo com o lado a ser operado, com o membro superior ipsilateral mantido inicialmente ao longo do corpo. Uma incisão em forma de $\mathrm{L}$ foi feita na pele e subcutâneo, com início na borda lateral do acrômio até o ângulo superomedial da escápula, curvou-se então rumo ao ângulo inferior, sobre a margem medial. Foi desenvolvido um plano de exposição entre o retalho de pele mais subcutâneo e a musculatura escapular. Para a exposição das estruturas, optou-se pela desinserção e pelo rebatimento da porção posterior do músculo deltoide (-Fig.1A). O intervalo entre os músculos infraespinal e redondo menor foi desenvolvido para que pudéssemos demarcar com alfinete um ponto de referência estático e imutável: o tubérculo inferior da glenoide - facilmente palpável nesse local ( - Fig. 1B).

Através de dissecção cuidadosa da região anterior do músculo infraespinal e redondo menor, foram então identificados seus respectivos nervos: supraescapular e ramo do axilar e demarcados com alfinete no ponto de sua penetração nos ventres de cada músculo (-Fig. 2 A e $\mathbf{- 2} \mathbf{B}$ ).

Foram feitas as medições, com o uso de um paquímetro universal, entre o ponto de referência estático definido - TI - e os alfinetes colocados nos pontos mais distais de cada nervo, quantificaram-se em milímetros as distâncias: TI ao NSE e TI ao NA.

Além dos dados específicos das medições, foram coletados os dados dos cadáveres estudados: sexo, idade, peso, altura.

Critérios de exclusão: espécimes que mostrassem sinais de lesões ou cirurgias prévias no ombro ou doenças prévias da cintura escapular.

\section{Resultados}

Os parâmetros foram avaliados em 13 ombros de 13 cadáveres frescos. Desses, oito eram do sexo masculino e cinco eram mulheres. A média foi de 70,1 anos (de 48-98). 


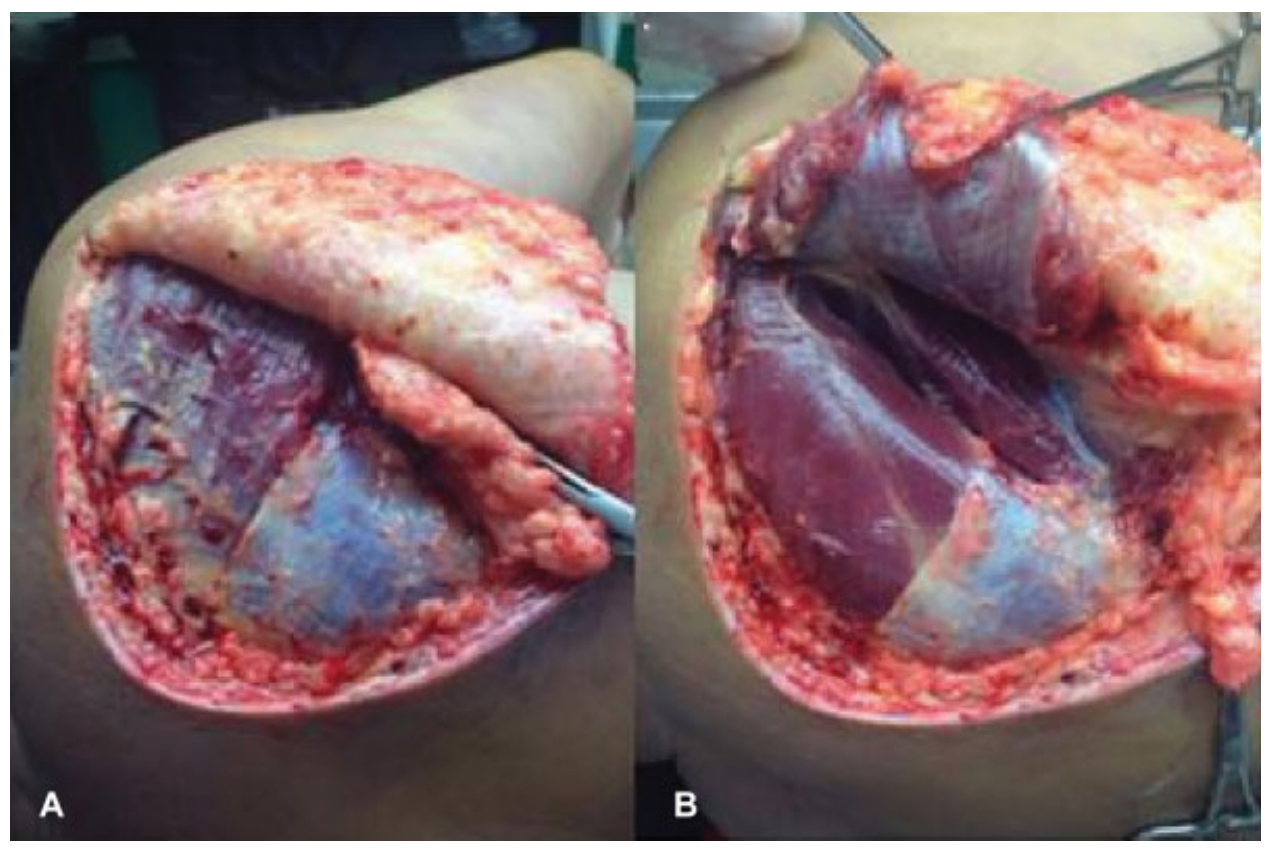

Fig. 1 Incisão em forma de L sobre borda medial da escapula e retalho subcutâneo que expõe a musculatura (A); rebatimento da porção posterior do músculo deltoide e divulsão do intervalo entre músculo infraespinal e redondo maior (B).

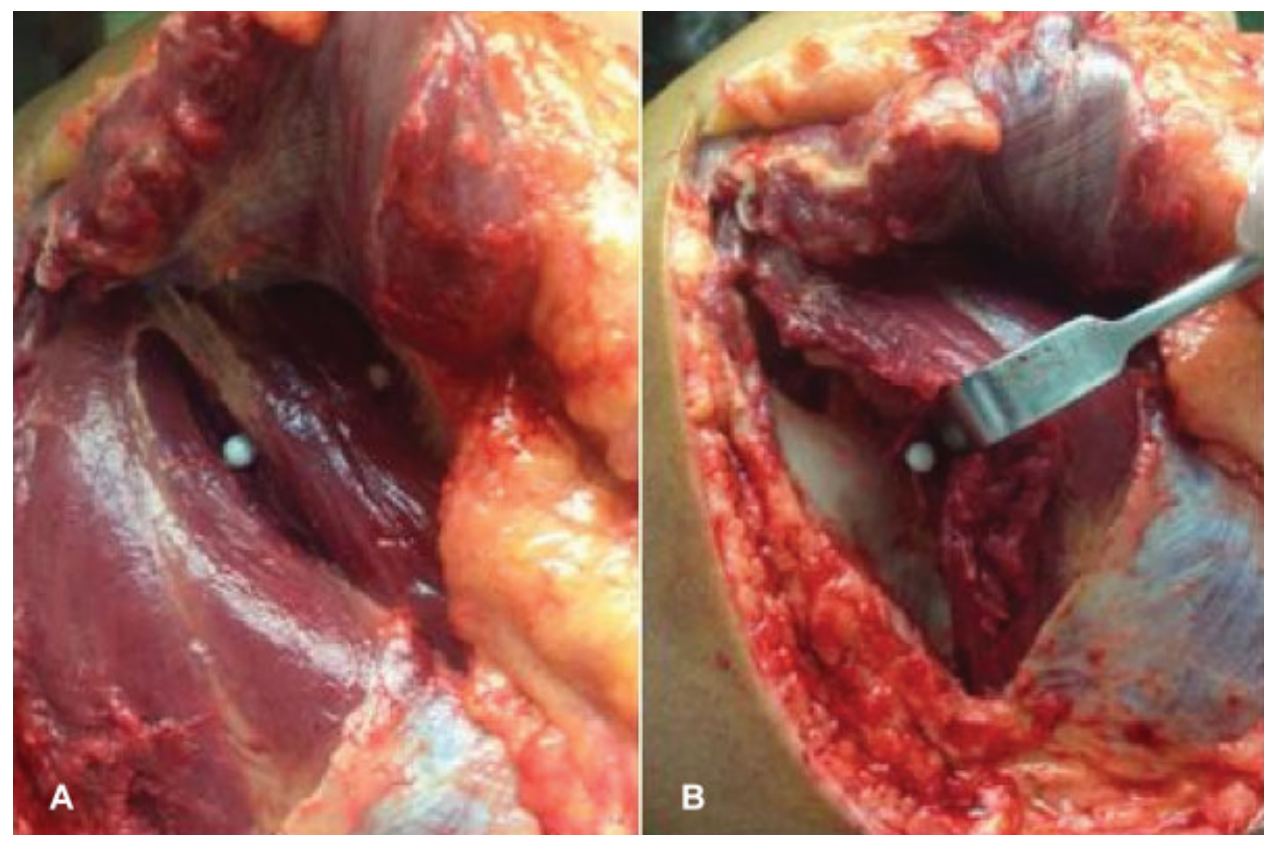

Fig. 2 Marcação com alfinete no tubérculo infraglenoidal no intervalo intermuscular e sobre porção mais distal do ramo do nervo axilar para o músculo redondo menor (A); marcação do nervo supraescapular sobre sua porção mais distal dissecada (B).

O peso médio dos cadáveres estudados foi de $61,5 \mathrm{~kg}$, enquanto que a altura média foi de $1,64 \mathrm{~m}$, variou de 1,52 a $1,75 \mathrm{~m}$. A distância média do TI ao NA foi de $23,8 \mathrm{~mm}$, variando de 17 a $28 \mathrm{~mm}$, e o desvio-padrão(DP) foi de 7,6 mm. A distância média do TI ao NSE foi de $33,2 \mathrm{~mm}$, variando de 17 a $43 \mathrm{~mm}$. 0 DP foi de $23,8 \mathrm{~mm}$, variando de 17 a $32 \mathrm{~mm}$.

\section{Discussão}

Com o aumento da incidência de fraturas de escápula devido aos traumas de grande energia, é natural que haja também aumento da gravidade dessas fraturas e, assim, da probabilidade de tratamento cirúrgico.

Barbieri et al. ${ }^{11}$ relataram bons resultados em 106 pacientes com fratura de escápula tratados conservadoramente e sugerem que os casos cirúrgicos são a minoria por ser, entre outras causas, uma abordagem difícil e com riscos de lesões musculares.

Mesmo com o desenvolvimento de novas técnicas de acesso, como visto nos trabalhos de Jerosch et al., ${ }^{8}$ Wirth et al. ${ }^{9}$ e Pizanis et al., ${ }^{12}$ que relataram baixa incidência de complicações, a abordagem cirúrgica da escápula pode colocar em risco algumas 
estruturas neurovasculares, como o NA e o NSE, com lesões ocorrendo em até 2 a 3\% dos pós-operatórios. A tração excessiva desses nervos pelo afastamento dos tecidos durante o acesso pode causar enfraquecimento do manguito rotador. ${ }^{6}$

Jerosch et al. desenvolveram um acesso posterior subdeltóideo e revelaram uma distância média de 21,98 $\mathrm{mm}$ até o NA, porém não estabeleceram em seu trabalho os pontos de referência usados. ${ }^{8}$

Longo et al. ${ }^{13}$ citaram em seu estudo uma taxa de $6 \%$ de lesão do NSE durante cirurgias para instabilidade do ombro e atentaram para o fato de o curso desse nervo estar alterado em casos de rupturas do manguito rotador. Durante seu estudo foi feita a medição da distância entre a borda posterior da glenoide e o NSE na incisura espinoglenoidal com valores médios de $12 \mathrm{~mm}$ em rotação interna e $19 \mathrm{~mm}$ em rotação externa do ombro.

Wirth et al. ${ }^{9}$ estudaram um acesso posterior através da divisão do músculo deltoide e aconselharam precaução com o NSE durante a dissecção, que pode estar situado cerca de $15 \mathrm{~mm}$ medial à borda da glenoide posterior. Ball et al., ${ }^{14} \mathrm{em}$ um estudo da anatomia do ramo posterior do NA, descreveram que o ramo para o músculo redondo menor surge imediatamente à borda inferior da glenoide junto à origem da cabeça longa do tríceps e mede cerca de 11 a $25 \mathrm{~mm}$ (média de $18 \mathrm{~mm}$ ) até sua entrada no músculo.

Consideramos que a origem do ramo do NA para o músculo redondo menor no estudo de Ball seja a mesma do nosso parâmetro usado para a medição, que é o TI. Assim, em comparação com o nosso estudo, atingimos valores ligeiramente maiores com média de $23,8 \mathrm{~mm}$ para a distância entre 0 TI e o NA.

Shaffer et al., ${ }^{15}$ durante avaliação anatomoeletroneuromiográfica do NSE durante um acesso posterior com divisão horizontal do músculo infraespinal, obtiveram um valor médio de 22,5 mm de distância entre a borda posterior da glenoide até o ramo mais próximo que atravessava essa divisão muscular. Essa medição tem os parâmetros bem próximos aos de nosso estudo referentes ao mesmo nervo, porém apresenta valores consideravelmente inferiores ao encontrados em nossa medição, cujo valor médio foi de 33,2 mm para a distância entre oTI e o NSE, provavelmente pela diferença de parâmetro ósseo e a divisão muscular ter sido aleatória no estudo de Shaffer.

É importante ressaltar que em nosso estudo a média de idade dos pacientes foi de 70,1 anos e o peso médio de 60,5 kg. Isso reflete o perfil dos espécimes idosos estudados em um serviço de verificação de óbito, com características musculares hipotróficas esperadas para a faixa etária estudada, e sua possível influência no valor das distâncias entre os parâmetros estabelecidos. Supomos que em indivíduos mais jovens e ativos os valores podem ser relativamente maiores devido a maior trofismo muscular. Porém, devido ao número reduzido de espécimes estudados, nos impossibilitamos de comprovar tal afirmação.

\section{Conclusão}

A abordagem cirúrgica para o tratamento das fraturas do colo da glenoide é considerada segura através do intervalo entre os músculos infraespinal e redondo menor, porém deve-se prestar atenção e ter cautela durante afastamento muscular devido à curta distância média entre o sítio de fratura e a localização do NSE e do NA, evitando assim maiores complicações pós-operatórias.

\section{Conflitos de Interesse}

Os autores declaram não haver conflitos de interesse.

\section{Referências}

1 Ada JR, Miller ME. Scapular fractures. Analysis of 113 cases. Clin Orthop Relat Res 1991;(269):174-180

2 McGahan JP, Rab GT, Dublin A. Fractures of the scapula. J Trauma 1980;20(10):880-883

3 Thompson DA, Flynn TC, Miller PW, Fischer RP. The significance of scapular fractures. J Trauma 1985;25(10):974-977

4 Geel CW. Scapula and clavicle. In: Colton CL, Fernandez DellO' ca A, Holz U, Kellam JF, Ochsner PE, eds. AO principles of fracture management. New York: Thieme: Stuttgart; 2000:261-2

5 Jones CB, Cornelius JP, Sietsema DL, Ringler JR, Endres TJ. Modified Judet approach and minifragment fixation of scapular body and glenoid neck fractures. J Orthop Trauma 2009;23(08):558-564

6 Cole PA, Dubin JR, Freeman G. Operative techniques in the management of scapular fractures. Orthop Clin North Am 2013; 44(03):331-343, viii

7 Salassa TE, Hill BW, Cole PA. Quantitative comparison of exposure for the posterior Judet approach to the scapula with and without deltoid takedown. J Shoulder Elbow Surg 2014;23(11): 1747-1752

8 Jerosch J, Greig M, Peuker ET, Filler TJ. The posterior subdeltoid approach: a modified access to the posterior glenohumeral joint. J Shoulder Elbow Surg 2001;10(03):265-268

9 Wirth MA, Butters KP, Rockwood CA Jr. The posterior deltoidsplitting approach to the shoulder. Clin Orthop Relat Res 1993; (296):92-98

10 van Noort A, van Loon CJ, Rijnberg WJ. Limited posterior approach for internal fixation of a glenoid fracture. Arch Orthop Trauma Surg 2004;124(02):140-144

11 Barbieri CH, Mazzer N, Mendonça FH, Damasceno LHF. Fraturas da escápula. Rev Bras Ortop 2001;36(07):245-254

12 Pizanis A, Tosounidis G, Braun C, Pohlemann T, Wirbel RJ. The posterior two-portal approach for reconstruction of scapula fractures: results of 39 patients. Injury 2013;44(11):1630-1635

13 Longo UG, Forriol F, Loppini M, et al. The safe zone for avoiding suprascapular nerve injury in bone block procedures for shoulder instability. A cadaveric study. Knee Surg Sports Traumatol Arthrosc 2015;23(05):1506-1510

14 Ball CM, Steger T, Galatz LM, Yamaguchi K. The posterior branch of the axillary nerve: an anatomic study. J Bone Joint Surg Am 2003; 85(08):1497-1501

15 Shaffer BS, Conway J, Jobe FW, Kvitne RS, Tibone JE. Infraspinatus muscle-splitting incision in posterior shoulder surgery. An anatomic and electromyographic study. Am J Sports Med 1994;22 (01):113-120 